\title{
Remarks on the distribution of the primitive roots of a prime
}

\author{
Shane Chern
}

\begin{abstract}
Let $\mathbb{F}_{p}$ be a finite field of size $p$ where $p$ is an odd prime. Let $f(x) \in \mathbb{F}_{p}[x]$ be a polynomial of positive degree $k$ that is not a $d$-th power in $\mathbb{F}_{p}[x]$ for all $d \mid p-1$. Furthermore, we require that $f(x)$ and $x$ are coprime. The main purpose of this paper is to give an estimate of the number of pairs $\left(\xi, \xi^{\alpha} f(\xi)\right)$ such that both $\xi$ and $\xi^{\alpha} f(\xi)$ are primitive roots of $p$ where $\alpha$ is a given integer. This answers a question of Han and Zhang.
\end{abstract}

Keywords. Primitive root, character sum, Weil bound.

2010MSC. Primary 11A07; Secondary 11L40.

\section{Introduction}

Let $a$ and $q$ be relatively prime integers, with $q \geq 1$. We know from the Euler-Fermat theorem that $a^{\phi}(q) \equiv 1 \bmod q$, where $\phi(q)$ is the Euler totient function. We say an integer $f$ is the exponent of $a$ modulo $q$ if $f$ is smallest positive integer such that $a^{f} \equiv 1 \bmod q$. If $f=\phi(q)$, then $a$ is called a primitive root of $q$. If $q$ has a primitive root $a$, then the group of the reduced residue classes $\bmod q$ is the cyclic group generated by the residue class $\hat{a}$. It is well-known that primitive roots exist only for the following moduli:

$$
q=1,2,4, p^{\alpha}, \text { and } 2 p^{\alpha},
$$

where $p$ is an odd prime and $\alpha \geq 1$. The reader may refer to Chapter 10 of T. M. Apostol's book [1] for detailed contents.

There has been a long history studying the distribution of the primitive roots of a prime. In a recent paper, D. Han and W. Zhang [3] considered the number of pairs $\left(\xi, m \xi^{k}+n \xi\right)$ such that both $\xi$ and $m \xi^{k}+n \xi$ are primitive roots of an odd prime $p$ where $m, n$ and $k$ are given integers with $k \neq 1$ and $(m n, p)=1$. The reader may also find some descriptions of other interesting problems on primitive roots such as the Golomb's conjecture in [3] and references therein. After presenting their main results, Han and Zhang proposed the following

Question 1.1. Let $\mathbb{F}_{p}$ be a finite field of size $p$ and $f(x)$ be an irreducible polynomial in $\mathbb{F}_{p}[x]$. Whether there exists a primitive element $\xi \in \mathbb{F}_{p}$ such that $f(\xi)$ is also a primitive element in $\mathbb{F}_{p}$ ?

In this paper, we let $f(x) \in \mathbb{F}_{p}[x]$ be a polynomial of positive degree $k$ that is not a $d$ th power in $\mathbb{F}_{p}[x]$ for all $d \mid p-1$. Furthermore, we require that $f(x)$ and $x$ are coprime. Let $\alpha$ be a given integer, we denote by $N(\alpha, f ; p)$ the number of pairs $\left(\xi, \xi^{\alpha} f(\xi)\right)$ such that both $\xi$ and $\xi^{\alpha} f(\xi)$ are primitive roots of $p$. Our result is 
Theorem 1.1. It holds that

$$
N(\alpha, f ; p)=(p-1-R(f))\left(\frac{\phi(p-1)}{p-1}\right)^{2}+\theta k 4^{\omega(p-1)} \sqrt{p}\left(\frac{\phi(p-1)}{p-1}\right)^{2},
$$

where $|\theta|<1, \omega(n)$ denotes the number of distinct prime divisors of $n$, and $R(f)$ denotes the number of distinct zeros of $f(x)$ in $\mathbb{F}_{p}$.

Now if we take $\alpha=0$ and $f(x)=x+1$, then we get the famous result on consecutive primitive roots obtained by J. Johnsen [4] and M. Szalay [5]. If we take

$$
\begin{cases}\alpha=1 \text { and } f(x)=m x^{k-1}+n & \text { if } k>1, \\ \alpha=k \text { and } f(x)=n x^{1-k}+m & \text { if } k<1,\end{cases}
$$

then we get Han and Zhang's result immediately.

Remark 1.1. We should mention that there is a minor mistake in Han and Zhang's result. In fact, they forgot to consider the zeros of $f(x)$ in $\mathbb{F}_{p}$. For example, if we choose $f(x)=x^{-1}+x=x^{-1}\left(x^{2}+1\right)$, then there are $1+(-1 \mid p)$ distinct zeros of $x^{2}+1$ in $\mathbb{F}_{p}$ where $(* \mid p)$ is the Legendre symbol. In this sense, the main term of $N\left(-1, x^{2}+1 ; p\right)$ (or their $N(-1,1,1, p)$ ) should be

$$
(p-2-(-1 \mid p))\left(\frac{\phi(p-1)}{p-1}\right)^{2}
$$

while not $\phi^{2}(p-1) /(p-1)$.

\section{Preliminary lemmas}

We first introduce the indicator function of primitive roots.

Lemma 2.1 (L. Carlitz [2, Lemma 2]). We have

$$
\frac{\phi(p-1)}{p-1} \sum_{d \mid p-1} \frac{\mu(d)}{\phi(d)} \sum_{\substack{\chi \bmod p \\ \text { ord } \chi=d}} \chi(n)= \begin{cases}1 & \text { if } n \text { is a primitive root of } p \\ 0 & \text { otherwise. }\end{cases}
$$

Here $\mu$ is the Möbius function, and ord $\chi$ denotes the order of a Dirichlet character $\chi \bmod p$, that is, the smallest positive integer $f$ such that $\chi^{f}=\chi_{0}$, the principal character modulo $p$.

The following famous Weil bound for character sums plays an important role in our proof.

Lemma 2.2 (A. Weil [7]). Let $\chi$ be a non-principal Dirichlet character modulo $p$ with order $d$. Suppose $f(x) \in \mathbb{F}_{p}[x]$ is a polynomial of positive degree $k$ that is not a $d$-th power in $\mathbb{F}_{p}[x]$. Then we have

$$
\left|\sum_{n=1}^{p-1} \chi(f(n))\right| \leq(k-1) \sqrt{p} .
$$

We also need the less-known extension of Weil bound obtained by D. Wan. 
Lemma 2.3 (D. Wan [6, Corollary 2.3]). Let $\chi_{1}, \chi_{2}, \ldots, \chi_{m}$ be non-principal Dirichlet characters modulo $p$ with orders $d_{1}, d_{2}, \ldots, d_{m}$, respectively. Suppose $f_{1}(x), f_{2}(x), \ldots$, $f_{m}(x) \in \mathbb{F}_{p}[x]$ are pairwise prime polynomials of positive degrees $k_{1}, k_{2}, \ldots, k_{m}$. Suppose also that $f_{i}(x)$ is not a $d_{i}$-th power in $\mathbb{F}_{p}[x]$ for all $i=1,2, \ldots, m$. Then we have

$$
\left|\sum_{n=1}^{p-1} \chi_{1}\left(f_{1}(n)\right) \chi_{2}\left(f_{2}(n)\right) \cdots \chi_{m}\left(f_{m}(n)\right)\right| \leq\left(\sum_{i=1}^{m} k_{i}-1\right) \sqrt{p} .
$$

From Lemmas 2.2 and 2.3, we have

Lemma 2.4. Let $\chi_{1}$ be a Dirichlet character modulo $p$, and $\chi_{2}$ be a non-principal Dirichlet character modulo $p$ with order $d$. Suppose $f(x) \in \mathbb{F}_{p}[x]$ is a polynomial of positive degree $k$ that is not a $d$-th power in $\mathbb{F}_{p}[x]$. We also require that $f(x)$ and $x$ are coprime. Furthermore, let $\alpha$ be a given integer. Then we have

$$
\left|\sum_{n=1}^{p-1} \chi_{1}\left(n^{\alpha}\right) \chi_{2}(f(n))\right| \leq \begin{cases}(k-1) \sqrt{p} & \text { if } \chi_{1}^{\alpha} \text { is the principal character } \\ k \sqrt{p} & \text { otherwise. }\end{cases}
$$

Proof. Note that

$$
\sum_{n=1}^{p-1} \chi_{1}\left(n^{\alpha}\right) \chi_{2}(f(n))=\sum_{n=1}^{p-1} \chi_{1}^{\alpha}(n) \chi_{2}(f(n)) .
$$

Now if $\chi_{1}^{\alpha}$ is the principal character, then it follows that

$$
\sum_{n=1}^{p-1} \chi_{1}\left(n^{\alpha}\right) \chi_{2}(f(n))=\sum_{n=1}^{p-1} \chi_{2}(f(n))
$$

and we get the bound from Lemma 2.2. If $\chi_{1}^{\alpha}$ is not the principal character, then the bound is obtained through a direct application of Lemma 2.3.

\section{Proof of the main result}

It follows by Lemma 2.1 that

$$
\begin{aligned}
& N(\alpha, f ; p) \\
& =\sum_{n=1}^{p-1}\left(\frac{\phi(p-1)}{p-1}\right)^{2} \sum_{d_{1} \mid p-1} \sum_{d_{2} \mid p-1} \frac{\mu\left(d_{1}\right)}{\phi\left(d_{1}\right)} \frac{\mu\left(d_{2}\right)}{\phi\left(d_{2}\right)} \sum_{\substack{\chi_{1} \bmod p \\
\text { ord } \chi_{1}=d_{1}}} \sum_{\substack{\chi_{2} \bmod p \\
\text { ord } \chi_{2}=d_{2}}} \chi_{1}(n) \chi_{2}\left(n^{\alpha} f(n)\right) \\
& =(p-1-R(f))\left(\frac{\phi(p-1)}{p-1}\right)^{2} \\
& \quad+\left(\frac{\phi(p-1)}{p-1}\right)^{2} \sum_{\substack{d_{1} \mid p-1 \\
d_{1}>1}} \frac{\mu\left(d_{1}\right)}{\phi\left(d_{1}\right)} \sum_{\substack{\chi_{1} \bmod p \\
\text { ord } \chi_{1}=d_{1}}} \sum_{n-1}^{p-1} \chi_{1}(n) \\
& +\left(\frac{\phi(p-1)}{p-1}\right)^{2} \sum_{\substack{d_{2} \mid p-1 \\
d_{2}>1}} \frac{\mu\left(d_{2}\right)}{\phi\left(d_{2}\right)} \sum_{\substack{\chi_{2} \bmod p \\
\text { ord } \chi_{2}=d_{2}}} \sum_{n-1}^{p-1} \chi_{2}\left(n^{\alpha} f(n)\right)
\end{aligned}
$$




$$
+\left(\frac{\phi(p-1)}{p-1}\right)^{2} \sum_{\substack{d_{1} \mid p-1 \\ d_{1}>1}} \sum_{\substack{d_{2} \mid p-1 \\ d_{2}>1}} \frac{\mu\left(d_{1}\right)}{\phi\left(d_{1}\right)} \frac{\mu\left(d_{2}\right)}{\phi\left(d_{2}\right)} \sum_{\substack{\chi_{1} \bmod p \\ \text { ord } \chi_{1}=d_{1}}} \sum_{\substack{\chi_{2} \bmod \chi_{2}=d_{2} \\ \text { ord }}} \sum_{n=1}^{p-1} \chi_{1}(n) \chi_{2}\left(n^{\alpha} f(n)\right) .
$$

Claim 3.1. We have

$$
\sum_{\substack{d_{1} \mid p-1 \\ d_{1}>1}} \frac{\mu\left(d_{1}\right)}{\phi\left(d_{1}\right)} \sum_{\substack{\chi_{1} \bmod p \\ \text { ord } \chi_{1}=d_{1}}} \sum_{n-1}^{p-1} \chi_{1}(n)=0
$$

Proof. We deduce it directly from

$$
\sum_{n=1}^{p-1} \chi(n)=0
$$

if $\chi$ is not the principal character modulo $p$.

Claim 3.2. We have

$$
\left|\sum_{\substack{d_{2} \mid p-1 \\ d_{2}>1}} \frac{\mu\left(d_{2}\right)}{\phi\left(d_{2}\right)} \sum_{\substack{\chi_{2} \bmod p \\ \text { ord } \chi_{2}=d_{2}}} \sum_{n-1}^{p-1} \chi_{2}\left(n^{\alpha} f(n)\right)\right| \leq\left(2^{\omega(p-1)}-1\right) k \sqrt{p} .
$$

Proof. Note that

$$
\sum_{n-1}^{p-1} \chi_{2}\left(n^{\alpha} f(n)\right)=\sum_{n=1}^{p-1} \chi_{2}\left(n^{\alpha}\right) \chi_{2}(f(n)) .
$$

Now by Lemma 2.4, we have

$$
\left|\sum_{n-1}^{p-1} \chi_{2}\left(n^{\alpha} f(n)\right)\right| \leq k \sqrt{p}
$$

Note also that

$$
\sum_{\substack{d \mid p-1 \\ d>1}}|\mu(d)|=2^{\omega(p-1)}-1 .
$$

We therefore have

$$
\begin{aligned}
\left|\sum_{\substack{d_{2} \mid p-1 \\
d_{2}>1}} \frac{\mu\left(d_{2}\right)}{\phi\left(d_{2}\right)} \sum_{\substack{\chi_{2} \bmod p \\
\text { ord } \chi_{2}=d_{2}}} \sum_{n-1}^{p-1} \chi_{2}\left(n^{\alpha} f(n)\right)\right| & \leq \sum_{\substack{d_{2} \mid p-1 \\
d_{2}>1}}\left|\frac{\mu\left(d_{2}\right)}{\phi\left(d_{2}\right)}\right| \sum_{\substack{\chi_{2} \bmod p \\
\text { ord } \chi_{2}=d_{2}}}\left|\sum_{n-1}^{p-1} \chi_{2}\left(n^{\alpha} f(n)\right)\right| \\
& \leq \sum_{\substack{d_{2} \mid p-1 \\
d_{2}>1}}\left|\frac{\mu\left(d_{2}\right)}{\phi\left(d_{2}\right)}\right| \phi\left(d_{2}\right) k \sqrt{p} \\
& =\left(2^{\omega(p-1)}-1\right) k \sqrt{p} .
\end{aligned}
$$


Claim 3.3. We have

$$
\left|\sum_{\substack{d_{1} \mid p-1 \\ d_{1}>1}} \sum_{\substack{d_{2} \mid p-1 \\ d_{2}>1}} \frac{\mu\left(d_{1}\right)}{\phi\left(d_{1}\right)} \frac{\mu\left(d_{2}\right)}{\phi\left(d_{2}\right)} \sum_{\substack{\chi_{1} \bmod p \\ \text { ord } \chi_{1}=d_{1}}} \sum_{\substack{\chi_{2} \bmod p \\ \text { ord } \chi_{2}=d_{2}}} \sum_{n=1}^{p-1} \chi_{1}(n) \chi_{2}\left(n^{\alpha} f(n)\right)\right| \leq\left(2^{\omega(p-1)}-1\right)^{2} k \sqrt{p} .
$$

Proof. Note that

$$
\sum_{n=1}^{p-1} \chi_{1}(n) \chi_{2}\left(n^{\alpha} f(n)\right)=\sum_{n=1}^{p-1} \chi_{1} \chi_{2}^{\alpha}(n) \chi_{2}(f(n)) .
$$

Again by Lemma 2.4, we get

$$
\left|\sum_{n=1}^{p-1} \chi_{1}(n) \chi_{2}\left(n^{\alpha} f(n)\right)\right| \leq k \sqrt{p}
$$

We therefore have

$$
\begin{aligned}
& \left|\sum_{\substack{d_{1} \mid p-1 \\
d_{1}>1}} \sum_{\substack{d_{2} \mid p-1 \\
d_{2}>1}} \frac{\mu\left(d_{1}\right)}{\phi\left(d_{1}\right)} \frac{\mu\left(d_{2}\right)}{\phi\left(d_{2}\right)} \sum_{\substack{\chi_{1} \bmod p \\
\text { ord } \chi_{1}=d_{1}}} \sum_{\substack{\chi_{2} \bmod p \\
\text { ord } \chi_{2}=d_{2}}} \sum_{n=1}^{p-1} \chi_{1}(n) \chi_{2}\left(n^{\alpha} f(n)\right)\right| \\
& \leq \sum_{\substack{d_{1} \mid p-1 \\
d_{1}>1}} \sum_{\substack{d_{2} \mid p-1 \\
d_{2}>1}}\left|\frac{\mu\left(d_{1}\right)}{\phi\left(d_{1}\right)}\right|\left|\frac{\mu\left(d_{2}\right)}{\phi\left(d_{2}\right)}\right| \sum_{\substack{\chi_{1} \bmod p \\
\text { ord } \chi_{1}=d_{1}}} \sum_{\substack{\chi_{2} \bmod p \\
\text { ord } \chi_{2}=d_{2}}}\left|\sum_{n=1}^{p-1} \chi_{1}(n) \chi_{2}\left(n^{\alpha} f(n)\right)\right| \\
& \leq \sum_{\substack{d_{1} \mid p-1 \\
d_{1}>1}} \sum_{d_{2} \mid p-1}\left|\frac{\mu\left(d_{1}\right)}{\phi\left(d_{1}\right)}\right|\left|\frac{\mu\left(d_{2}\right)}{\phi\left(d_{2}\right)}\right| \phi\left(d_{1}\right) \phi\left(d_{2}\right) k \sqrt{p} \\
& =\left(2^{\omega(p-1)}-1\right)^{2} k \sqrt{p} .
\end{aligned}
$$

We conclude by combining Claims 3.1-3.3 that

$$
\begin{aligned}
& \left|N(\alpha, f ; p)-(p-1-R(f))\left(\frac{\phi(p-1)}{p-1}\right)^{2}\right| \\
& \quad \leq\left(\left(2^{\omega(p-1)}-1\right)+\left(2^{\omega(p-1)}-1\right)^{2}\right) k \sqrt{p}\left(\frac{\phi(p-1)}{p-1}\right)^{2} \\
& \quad<k 4^{\omega(p-1)} \sqrt{p}\left(\frac{\phi(p-1)}{p-1}\right)^{2} .
\end{aligned}
$$

This completes our proof.

\section{References}

1. T. M. Apostol, Introduction to analytic number theory, Undergraduate Texts in Mathematics, Springer-Verlag, New York-Heidelberg, 1976. xii+338 pp.

2. L. Carlitz, Sets of primitive roots, Compositio Math. 13 (1956), 65-70.

3. D. Han and W. Zhang, On the existence of some special primitive roots mod p, Bull. Math. Soc. Sci. Math. Roumanie (N.S.) 58(106) (2015), no. 1, 59-66. 
4. J. Johnsen, On the distribution of powers in finite fields, J. Reine Angew. Math. 251 (1971), 10-19. 5. M. Szalay, On the distribution of the primitive roots of a prime, J. Number Theory 7 (1975), 184-188.

6. D. Wan, Generators and irreducible polynomials over finite fields, Math. Comp. 66 (1997), no. 219, 1195-1212.

7. A. Weil, On some exponential sums, Proc. Nat. Acad. Sci. U. S. A. 34 (1948), 204-207.

School of Mathematical Sciences, Zhejiang University, Hangzhou, 310027, China

E-mail address: shanechern@zju.edu.cn; chenxiaohang92@gmail.com 